\title{
Unitary units in modular group algebras
}

\author{
Victor Bovdi and L. G. Kovács
}

Let $p$ be a prime, $K$ a field of characteristic $p, G$ a locally finite $p$-group, $K G$ the group algebra, and $V$ the group of the units of $K G$ with augmentation 1 . The anti-automorphism $g \mapsto g^{-1}$ of $G$ extends linearly to $K G$; this extension leaves $V$ setwise invariant, and its restriction to $V$ followed by $v \mapsto v^{-1}$ gives an automorphism of $V$. The elements of $V$ fixed by this automorphism are called unitary; they form a subgroup. Our first theorem describes the $K$ and $G$ for which this subgroup is normal in $V$.

For each element $g$ in $G$, let $\bar{g}$ denote the sum (in $K G$ ) of the distinct powers of $g$. The elements $1+(g-1) h \bar{g}$ with $g, h \in G$ are the bicyclic units of $K G$. Our second theorem describes the $K$ and $G$ for which all bicyclic units are unitary.

\section{Introduction}

Let $K G$ be the group algebra of a group $G$ over a commutative ring $K$ (with 1 ) and $V(K G)$ the group of normalized units (that is, of the units with augmentation 1 ) in $K G$. The anti-automorphism $g \mapsto g^{-1}$ extends linearly to an anti-automorphism $a \mapsto a^{*}$ of $K G$; this extension leaves $V(K G)$ setwise invariant, and its restriction to $V(K G)$ followed by $v \mapsto v^{-1}$ gives an automorphism of $V(K G)$. The elements of $V(K G)$ fixed by this automorphism are the unitary normalized units of $K G$; they form a subgroup which we denote by $V_{*}(K G)$. (Interest in unitary units arose in algebraic topology, and a more general definition, involving an 'orientation homomorphism', is also current; the special case we use here arises when the orientation homomorphism is trivial.)

The first question considered here is to find the pairs $K, G$ for which $V_{*}(K G)$ is normal in $V(K G)$. (Since each unit of a group algebra is a scalar multiple of a normalized unit, if $V_{*}(K G)$ is normal in $V(K G)$ then it is normal also in the group of all units of $K G$.) For $K=\mathbb{Z}$, this question was discussed (without any restriction on the orientation homomorphism) by A. A. Bovdi and S. K. Sehgal in [4]. Here we deal with the 'modular' case, that is, with the case of $K$ a field of prime characteristic $p$ and $G$ a locally finite $p$-group.

Theorem 1.1. Let $K$ be a field of prime characteristic $p$ and let $G$ be a nonabelian locally finite $p$-group. The subgroup $V_{*}(K G)$ is normal in $V(K G)$ if and only if $p=2$ and $G$ is the direct product of an elementary abelian group with a group $H$ for which one of the following holds:

(i) $H$ has no direct factor of order 2, but it is a semidirect product of a group $\langle h\rangle$ of order 2 and an abelian 2 -group $A$, with $h^{-1} a h=a^{-1}$ for all a in $A$;

(ii) $H$ is an extraspecial 2-group, or the central product of such a group with a cyclic group of order 4 .

We work with the definition that a $p$-group is extraspecial if its centre, commutator subgroup and Frattini subgroup are equal and have order $p$ : we do not require the group itself to be finite.

The proof of this theorem will be given in Section 2. The reason we take the $p$-group $G$ locally finite is that, as is well known, this ensures that each non-unit of $K G$ lies in the augmentation ideal.

Research partly supported by the Hungarian National Foundation for Scientific Research grant no. T4265.

The second author is indebted to the 'Universitas' Foundation and the Lajos Kossuth University of Debrecen, Hungary, for warm hospitality and generous support during the period when this work began. 
Every group $G$ may be written (see Lemma 2.3 ) as a direct product of an elementary abelian 2-group $E$ and a group $H$ which has no direct factor of order 2 (we do not exclude $E=1$ or $H=1$ ). The isomorphism type of $G$ determines the isomorphism types of $E$ and $H$, and vice versa.

It is easy to verify that if $H$ satisfies (i) then $A=\left\langle a \in H \mid a^{2} \neq 1\right\rangle$ and $A$ has no direct factor of order 2. Conversely, if $A$ is a nontrivial abelian 2-group without a direct factor of order 2 and $H$ is formed as the semidirect product indicated, then (i) holds. The classification of the groups $H$ of this kind is thus reduced to the classification of abelian 2-groups, a problem whose solution in terms of Ulm invariants is well known in the finite or countably infinite case but is beyond reach in general.

As to case (ii), the classification of finite extraspecial groups is well known. Equally conclusive results were obtained for extraspecial groups of countably infinite order by M. F. Newman in [9]; he also showed there that no such results can be expected for extraspecial groups of arbitrary order.

The only group $H$ which satisfies both conditions (i) and (ii) is the dihedral group of order 8 .

The second part of the paper concerns the bicyclic units introduced in Ritter and Sehgal [11]. For $K$ a commutative ring and $g$ an element of finite order $|g|$ in a group $G$, let $\bar{g}$ denote the sum (in $K G$ ) of the distinct powers of $g$ :

$$
\bar{g}=\sum_{i=0}^{|g|-1} g^{i} .
$$

If also $h \in G$, put

$$
u_{g, h}=1+(g-1) h \bar{g}
$$

Note that $1-(g-1) h \bar{g}$ is a two-sided inverse for $u_{g, h}$ and the augmentation of $u_{g, h}$ is 1 , so $u_{g, h}$ is a normalized unit. The elements of this form are called bicyclic units.

The problem considered here is to find the $K$ and $G$ for which each bicyclic unit of $K G$ is unitary. It is easy to see that $u_{g, h}=1$ if and only if the cyclic group $\langle g\rangle$ is normalized by $h$, and that if $K=\mathbb{Z}$ then 1 is the only bicyclic unit which is unitary. Thus the $G$ which can partner $K=\mathbb{Z}$ are precisely the groups in which every subgroup of finite order is normal. (The situation is not so simple for $K=\mathbb{Z}$ when unitarity is defined with reference to a nontrivial orientation homomorphism: see A. A. Bovdi and S. K. Sehgal [3].) In private communication, Professor Sehgal has directed attention to the modular case: what can one say when $K$ is of characteristic $p$ and $G$ is a $p$-group?

Theorem 1.2. Let $p$ be a prime, $K$ a commutative ring of characteristic $p$, and $G$ a nonabelian $p$-group. All bicyclic units of $K G$ are unitary if and only if $p=2$ and $G$ is the direct product of an elementary abelian group and a group $H$ for which one of the following holds:

(i) $H$ has an abelian subgroup $A$ of index 2 such that conjugation by an element of $H$ outside $A$ inverts each element of $A$;

(ii) $H$ is an extraspecial 2 -group, or the central product of such a group with a cyclic group of order 4;

(iii) $H$ is the direct product of a quaternion group of order 8 and a cyclic group of order 4 , or the direct product of two quaternion groups of order 8;

(iv) $H$ is the central product of the group $\left\langle x, y \mid x^{4}=y^{4}=1, x^{2}=[y, x]\right\rangle$ with a quaternion group of order 8 , the nontrivial element common to the two central factors being $x^{2} y^{2}$;

(v) $H$ is isomorphic to one of the groups $H_{32}$ and $H_{245}$ defined below.

The relevant definitions are:

$$
\begin{gathered}
H_{32}=\langle x, y, u| x^{4}=y^{4}=1, \\
x^{2}=[y, x], \\
y^{2}=u^{2}=[u, x], \\
\left.x^{2} y^{2}=[u, y]\right\rangle, \\
H_{245}=\langle x, y, u, v| x^{4}=y^{4}=[v, u]=1, \\
x^{2}=v^{2}=[y, x]=[v, y], \\
y^{2}=u^{2}=[u, x], \\
\left.x^{2} y^{2}=[u, y]=[v, x]\right\rangle .
\end{gathered}
$$


The subscripts are the serial numbers of these groups in the CAYLEY library of groups of order dividing 128 described by Newman and O'Brien in [10]. It is a mere coincidence that $H_{32}$ has order 32 . The other group, $H_{245}$, is one of the two Suzuki 2-groups (see Higman [7]) of order 64. That CAYLEY library provides us not only with serial numbers but also with the final step in the proof of Theorem 1.2: we are indebted to Dr E. A. O'Brien for extracting the list (Lemma 4.1) of the groups $H$ of order dividing 128 whose Frattini subgroup is central, noncyclic, of order 4 , and contains all elements of order 2 in $H$. His list shows that all groups of this kind have order dividing 64, so they may also be found in Hall and Senior [6], where $H_{32}$ and $H_{245}$ are labelled $32 \Gamma_{4} c_{3}$ and $64 \Gamma_{13} a_{5}$. (Of course, without the much larger CAYLEY library we could not see that no group of order 128 satisfies these criteria.)

Several of the comments we made after Theorem 1.1 apply here as well. In (i) we can assume that $H$ has no direct factor of order 2: the isomorphism type of $H$ is then determined by $G$. We may also assume there that $|H|>8$, for the two nonabelian groups of order 8 occur also under (ii). It is easy to see that then $H$ has only one abelian subgroup of index 2, so the isomorphism type of $A$ is in turn determined by $H$. Moreover, the squares of all the elements of $H$ outside $A$ are equal to each other, and this element, $a_{0}$ say, has order at most 2. Of course the height of $a_{0}$ in $A$ is also an isomorphism invariant of $H$. Mackey's proof of Ulm's Theorem (given in Kaplansky [8]) shows that if two countable $p$-groups have the same Ulm invariants and we are given a height-preserving isomorphism from a finite subgroup of one to a subgroup of the other, then this will extend to an isomorphism of the two groups. It follows that in the finite or countably infinite case the Ulm invariants of $A$ together with the height in $A$ of the common square of the elements outside $A$ form a complete set of invariants for $H$. Conversely, if $a_{0}$ is any element of order at most 2 in an abelian 2-group $A$ with $|A|>4$, then the group $H$ defined by

$$
\left.H=\langle A, h| h^{2}=a_{0}, h^{-1} a h=a^{-1} \text { for all } a \text { in } A\right\rangle
$$

satisfies (i) and $|H|>8$. (The reader may like to work out how the relevant invariants must be restricted to ensure that $H$ is nonabelian and has no direct factor of order 2.)

The proof of Theorem 1.2 splits naturally into a ring-theoretic part and a group-theoretic part, which are presented in Section 3 and Section 4. Their conclusions make sense separately and may be of some independent interest, so we state them here.

Lemma 1.3. Let $K$ be a commutative ring with 1 and $G$ a group. Suppose that $g$ is an element of finite order in $G$ and $h$ is an element of $G$ which does not normalize $\langle g\rangle$. The bicyclic unit $u_{g, h}$ is unitary if and only if the characteristic of $K$ is 2 while $\left\langle g^{2}\right\rangle$ is normalized by $h$ and contains either $h^{2}$ or $(h g)^{2}$.

(In particular, this confirms the comment above that in $\mathbb{Z} G$ the only unitary bicyclic unit is 1 . In fact, $V_{*}(\mathbb{Z} G)$ is always $G$ itself, as was observed in Lemma 1 of A. A. Bovdi [1].)

Lemma 1.4. Let $G$ be a nonabelian 2 -group such that if $g, h \in G$ then $\left\langle g^{2}\right\rangle$ is normal in $G$ and $\langle g, h\rangle /\left\langle g^{2}\right\rangle$ is either abelian or dihedral. Then $G$ is the direct product of an elementary abelian group with a nonabelian group $H$ for which one of the conditions (i)-(v) of Theorem 1.2 holds. Conversely, every 2 -group $G$ of this kind satisfies our hypotheses.

\section{Normal unitary subgroup}

The aim of this section is to prove Theorem 1.1. It will be convenient to use $y^{*}=y^{-1}$ as the test of whether a unit $y$ is unitary. At first, $K$ can be any commutative ring with 1 and $G$ any group.

Lemma 2.1. For $x \in V(K G)$ and $y \in V_{*}(K G)$, we have $x^{-1} y x \in V_{*}(K G)$ if and only if $x x^{*}$ commutes with $y$.

Proof. Clearly, $\left(x^{-1} y x\right)^{*}=\left(x^{-1} y x\right)^{-1}$ means that $x^{*} y^{*}\left(x^{*}\right)^{-1}=x^{-1} y^{-1} x$ which in turn is equivalent to $x x^{*} y^{*}=y^{-1} x x^{*}$. As we are given that $y^{*}=y^{-1}$, this proves the lemma. 
As $G \leq V_{*}(K G)$, an element which commutes with every element of $V_{*}(K G)$ is central in $K G$. Thus Lemma 2.1 gives the following.

Corollary 2.2. The subgroup $V_{*}(K G)$ is normal in $V(K G)$ if and only if all elements of the form $x x^{*}$ with $x \in V(K G)$ are central in $K G$.

(For the case of $K=\mathbb{Z}$, this is a special case of Lemma 2 of [4]; the proof we have given comes from that paper.)

Proof of Theorem 1.1. From the simple fact that over a field of characteristic $p$ a finite $p$-group has only one irreducible representation, it follows readily that under the hypotheses of the theorem the augmentation ideal of $K G$ is locally nilpotent and so each element outside that ideal is a unit. Differently put, if $x$ is a non-unit in $K G$ then $1+K G \in V(K G)$.

Suppose first that $V_{*}(K G)$ is normal in $V(K G)$. By Corollary 1 , if $x$ is a normalized unit then $x x^{*}$ is central. As each unit is a scalar multiple of a normalized unit, the same conclusion is available whenever $x$ is a unit. It follows that if $x$ is any unit then $x x^{*}=x^{-1}\left(x x^{*}\right) x=x^{*} x$. If $x$ is a non-unit in $K G$, then $1+x$ is a unit and so $(1+x)(1+x)^{*}=(1+x)^{*}(1+x)$, whence again $x x^{*}=x^{*} x$.

A group algebra in which $x x^{*}=x^{*} x$ holds for every element $x$ is called normal. A. A. Bovdi, P.M. Gudivok and M. S. Semirot proved in [2] that the group algebra of a nonabelian group $G$ over a commutative ring $K$ is normal if and only if either $G$ is hamiltonian or the characteristic of $K$ is 2 and $G$ is a direct product of an elementary abelian 2 -group with a group $H$ such that (i) or (ii) holds. Thus the proof of our 'only if' claim is complete.

Suppose next that $p=2$ and $G=E \times H$ with $E$ elementary abelian and $H$ satisfying (i) or (ii). In view Corollary 2.2, what we have to show is that $x x^{*}$ is central whenever $x \in V(K G)$.

Consider first the case (i). Then each element $x$ of $K G$ can be written as $x=x_{1}+x_{2} h$ with $x_{1}, x_{2} \in K(E \times A)$, and of course $h x_{i} h=x_{i}^{*}$. Using again that $h^{2}=1$, that $K(E \times A)$ is commutative, and that the characteristic is 2 , we see that $x x^{*}=\left(x_{1}+x_{2} h\right)\left(x_{1}^{*}+h x_{2}^{*}\right)=x_{1} x_{1}^{*}+2 x_{1} x_{2} h+x_{2} x_{2}^{*}=x_{1} x_{1}^{*}+x_{2} x_{2}^{*}$. Thus $x x^{*}$ lies in the commutative algebra $K(E \times H)$ and is easily seen to commute with $h$, so it is central in $K G$.

Consider next the case (ii). Then the commutator subgroup of $G$ has only one nontrivial element; call that $c$, and write $I$ for the ideal of $K G$ generated by $1+c$. This element $c$ is central in $G$, while if $g, h \in G$ then either $h g=g h$ or $h g=g h c$ : so either $h g(1+c)=g h(1+c)=g(1+c) h$ or $h g(1+c)=g h c(1+c)=g h(1+c)=g(1+c) h$ proves that $g(1+c)$ commutes with $h$. It follows that every element of $I$, and therefore also every element of $1+I$, is central in $K G$.

Let $\gamma$ be the natural homomorphism of $K G$ onto $K(G /\langle c\rangle)$ defined by $g \gamma=g\langle c\rangle$ for all $g$ in $G$. Of course $\gamma$ intertwines the augmentation maps of the two group algebras, so if $x$ is a normalized unit in $K G$ then $x \gamma$ is a normalized unit in $K(G /\langle c\rangle)$. Further, $\gamma$ intertwines the anti-automorphism $*$ of $K G$ with the similarly defined anti-automorphism of $K(G /\langle c\rangle)$; we shall use $*$ also for the latter anti-automorphism. Note that $K(G /\langle c\rangle)$ is elementary abelian. It is an easy exercise to see that in a characteristic 2 group algebra of an elementary abelian 2-group each normalized unit is unitary. In particular, if $x \in V(K G)$ then $x \gamma$ is unitary, so $\left(x x^{*}\right) \gamma=(x \gamma)\left(x^{*} \gamma\right)=(x \gamma)(x \gamma)^{*}=1$, that is, $x x^{*} \in 1+\operatorname{ker} \gamma$. Since $I$ is minimal among the ideals for which $c \equiv 1 \bmod I$, it is precisely ker $\gamma$. We have proved that $x x^{*} \in 1+I$. By the conclusion of the previous paragraph, $x x^{*}$ is therefore central in $K G$. The proof of the theorem is now complete.

Remarks. On any group algebra of an elementary abelian 2-group, the anti-automorphism $*$ is in fact just the identity map.

For a generalization of the result of [2], see [5].

We conclude this section with a purely group-theoretic lemma which was mentioned in the introduction's comments on Theorem 1.1.

Lemma 2.3. Every group $G$ is a direct product $E \times H$ of an elementary abelian 2-group $E$ and a group $H$ which has no direct factor of order 2 . If $G=E_{1} \times H_{1}$ is another such decomposition of $G$, then $E_{1} \cong E$ and $H_{1} \cong H$. 
Proof. Let $\mathrm{Z}(G)$ denote the centre of $G$; set $A=\mathrm{A}(G)=\left\langle a \in \mathrm{Z}(G) \mid a^{2}=1\right\rangle$ and $B=\mathrm{B}(G)=\left\langle g^{2} \mid g \in G\right\rangle$. Let $E$ be a direct complement to $A \cap B$ in $A$, and $H / B$ a direct complement to $A B / B$ in $G / B$ : then

$$
G=A B H=E B H=E H \quad \text { while } \quad E \cap H \leq E \cap A B \cap H=E \cap B=1,
$$

so $G=E \times H$. Here $E$ is elementary abelian because $A$ is. If $H=C \times K$ with $|C| \leq 2$, then $G=E \times C \times K$ and $B=\mathrm{B}(K)$, so $C \leq A=E \times(A \cap B) \leq E \times K$ yields that $C=1$. This proves that $H$ has no direct factor of order 2 , that is, $\mathrm{A}(H) \leq \mathrm{B}(H)$. If $G=E_{1} \times H_{1}$ is another decomposition with $E_{1}$ elementary abelian and $\mathrm{A}\left(H_{1}\right) \leq \mathrm{B}\left(H_{1}\right)$, then $E_{1} \cong A /(A \cap B) \cong E$, and $H_{1} / B$ is another direct complement to $A B / B$ in $G / B$ : so we also have $G=E \times H_{1}$ and therefore $H_{1} \cong G / E \cong H$.

\section{Unitary bicyclic units}

The aim of this section is to prove Lemma 1.3. Accordingly, $K$ is once again an arbitrary commutative ring with 1 and $G$ is an arbitrary group. Recall the definition $u_{g, h}=1+(g-1) h \bar{g}$, and note that $u_{g, h}=u_{g, h g} \in V(K G)$, with

$$
u_{g, h}^{-1}=1-(g-1) h \bar{g} \quad \text { and } \quad u_{g, h}^{*}=1+\bar{g} h^{-1}\left(g^{-1}-1\right) .
$$

The support of an element $a$ of $K G$ is the set of those elements of $G$ which occur with nonzero coefficient in the expression of $a$ as $K$-linear combination of elements of $G$ :

$$
\operatorname{supp} \sum_{g \in G} \alpha_{g} g=\left\{g \in G \mid \alpha_{g} \neq 0\right\} .
$$

Two simple observations about bicyclic units will be used without reference. First, if $h$ normalizes $\langle g\rangle$ then $h \bar{g}=\bar{g} h$ and so $u_{g, h}=1$. Second, if $h$ does not normalize $\langle g\rangle$ then $u_{g, h} \neq 1$; indeed, in this case no element of $G$ can occur more than once in the expansion of $1+(g-1) h \bar{g}$, so the support of $u_{g, h}$ has cardinality $1+|g|+|g|$. Explicitly, if $h \notin \mathrm{N}(\langle g\rangle)$ then

$$
\operatorname{supp} u_{g, h}=\{1\} \cup\left\{g h g^{i}|0 \leq i<| g \mid\right\} \cup\left\{h g^{i}|0 \leq i<| g \mid\right\} .
$$

Proof of Lemma 1.3. Suppose that $u_{g, h}^{*}=u_{g, h}^{-1}$. If the characteristic of $K$ were not 2 , we could argue that in the expression of $u_{g, h}^{-1}$ as $1-(g-1) h \bar{g}$ both $h$ and $h g$ have coefficient 1 while in that of $u_{g, h}^{*}$ the only nontrivial elements of $G$ with coefficient 1 are the $g^{i} h^{-1} g^{-1}$, hence there exist $i, j$ such that $h=g^{i} h^{-1} g^{-1}$ and $h g=g^{j} h^{-1} g^{-1}$, and then

$$
h g h^{-1}=\left(g^{j} h^{-1} g^{-1}\right)\left(g^{i} h^{-1} g^{-1}\right)^{-1}=g^{j-i} \in\langle g\rangle
$$

contradicts the assumption that $h \notin \mathrm{N}(\langle g\rangle)$. Thus the characteristic of $K$ is 2 .

Note that $\left|g^{2}\right|$ can only be $|g|$ or $|g| / 2$. We exploit this repeatedly, for it yields that once we show $h^{-1} g^{2} h \in\langle g\rangle$, it follows that $h$ normalizes $\left\langle g^{2}\right\rangle$. Namely, if $h^{-1} g^{2} h \in\langle g\rangle$ then $h \notin \mathrm{N}(\langle g\rangle)$ implies that $\left\langle h^{-1} g^{2} h\right\rangle<\langle g\rangle$, whence $\left|h^{-1} g^{2} h\right|=\left|g^{2}\right|=|g| / 2$, and then $\left\langle h^{-1} g^{2} h\right\rangle$ must be the unique subgroup, $\left\langle g^{2}\right\rangle$, of index 2 in $\langle g\rangle$.

Since $u_{g, h} \neq 1$, the support of $u_{g, h}^{-1}$ is given by

$$
\operatorname{supp} u_{g, h}^{-1}=\{1\} \cup\left\{g h g^{i}|0 \leq i<| g \mid\right\} \cup\left\{h g^{i}|0 \leq i<| g \mid\right\}
$$

while

$$
\operatorname{supp} u_{g, h}^{*}=\{1\} \cup\left\{g^{i} h^{-1} g^{-1}|0 \leq i<| g \mid\right\} \cup\left\{g^{i} h^{-1}|0 \leq i<| g \mid\right\} .
$$

Given our assumption that $u_{g, h}^{*}=u_{g, h}^{-1}$, these two supports are equal. We now distinguish a number of cases according to the form in which various elements of $\operatorname{supp} u_{g, h}^{-1}$ appear in $\operatorname{supp} u_{g, h}^{*}$.

Suppose first that $h=g^{i} h^{-1}$, so $g h g=g^{i+1} h^{-1} g$. If $g h g=g^{j} h^{-1}$, then $h^{-1} g h=g^{j-i-1} \in\langle g\rangle$, contrary to $h \notin \mathrm{N}(\langle g\rangle)$. Thus $g h g=g^{j} h^{-1} g^{-1}$, and then $h^{-1} g^{2} h=g^{j-i-1} \in\langle g\rangle$, so $h$ normalizes $\left\langle g^{2}\right\rangle$. Of course now $h^{2}=g^{i} \in\langle g\rangle$, so $h^{2} \notin\left\langle g^{2}\right\rangle$ would imply that $\langle g\rangle=\left\langle\left\langle g^{2}\right\rangle, h^{2}\right\rangle$, which is impossible because $h \in \mathrm{N}\left(\left\langle g^{2}\right\rangle\right)$ but $h \notin \mathrm{N}(\langle g\rangle)$. This proves that in this case $h^{2} \in\left\langle g^{2}\right\rangle$. 
Suppose next that $h=g^{i} h^{-1} g^{-1}$, and note that $h \notin \mathrm{N}(\langle g\rangle)$ implies that $i \neq 0$. If $|g|=2$ then this forces $i=1$, so conjugation by $g$ inverts $h$ and therefore $(h g)^{2}=1$. Suppose that $|g|>2$; then $g^{i} h^{-1} g=h g^{2} \in \operatorname{supp} u_{g, h}^{-1}$. If $h g^{2}=g^{j} h^{-1}$, then $h^{-1} g h=g^{j-i} \in\langle g\rangle$, contrary to $h \notin \mathrm{N}(\langle g\rangle)$. Thus $h g^{2}=g^{j} h^{-1} g^{-1}$, and then $h^{-1} g^{2} h=g^{j-i} \in\langle g\rangle$, so $h$ normalizes $\left\langle g^{2}\right\rangle$. If $i$ is even, then in the factor group $\langle g, h\rangle /\left\langle g^{2}\right\rangle$ the image of $g$ is the square of the image of $h$, but this is impossible because $h \notin \mathrm{N}(\langle g\rangle)$. So $i$ is odd, and then in $\langle g, h\rangle /\left\langle g^{2}\right\rangle$ conjugation by the image of $g$ inverts the image of $h$ and therefore the image of $h g$ has order 2: thus again $(h g)^{2} \in\left\langle g^{2}\right\rangle$.

This completes the proof of the 'only if' claim. For the proof of the 'if' claim, assume first that the characteristic of $K$ is 2 , and note that then each bicyclic unit of $K G$ is its own inverse. Next, assume that $\left\langle g^{2}\right\rangle$ is normalized by $h$ and contains either $h^{2}$ or $(h g)^{2}$. Since in any case $u_{g, h}=u_{g, h g}$, we may assume without loss of generality that in fact $h^{2} \in\left\langle g^{2}\right\rangle$. Since $\left\langle g^{2}\right\rangle$ is normalized by $h$ but $\langle g\rangle$ is not, $|g|$ must be even, whence

$$
\bar{g}=(g+1) \overline{g^{2}}=\overline{g^{2}}\left(g^{-1}+1\right) .
$$

Further, as both $g$ and $h$ normalize $\left\langle g^{2}\right\rangle$, both commute with $\overline{g^{2}}$, while $h^{2} \in\left\langle g^{2}\right\rangle$ implies that $h^{2} \overline{g^{2}}=\overline{g^{2}}$ and so

$$
h \overline{g^{2}}=\overline{g^{2}} h^{-1} .
$$

Using again that the characteristic of $K$ is 2 , we can therefore argue that

$$
\begin{aligned}
u_{g, h}^{-1}=u_{g, h} & =1+(g+1) h \bar{g} \\
& =1+(g+1) h \overline{g^{2}}\left(g^{-1}+1\right) \\
& =1+(g+1) \overline{g^{2}} h^{-1}\left(g^{-1}+1\right) \\
& =1+\bar{g} h^{-1}\left(g^{-1}+1\right) \\
& =u_{g, h}^{*},
\end{aligned}
$$

as required.

\section{A certain class of groups}

The rest of the paper will be taken up by the proof of Lemma 1.4.

As usual, the Frattini subgroup of a group $H$ will be written $\Phi(H)$. Recall that if $H$ is a finite 2-group then $\Phi(H)=\left\langle h^{2} \mid h \in H\right\rangle$. If $H$ is any 2 -group, we write $\Omega(H)=\left\langle h \in H \mid h^{2}=1\right\rangle$. The proof of Lemma 1.4 depends on a result obtained for us by Dr E. A. O'Brien by inspecting the CAYLEY library described in Newman and O'Brien [10].

Lemma 4.1 (O'Brien). The groups $H$ of order dividing 128 in which $\Phi(H)$ and $\Omega(H)$ are equal, central, and of order 4 , are precisely the following: $C_{4} \times C_{4}, C_{4} \rtimes C_{4}, C_{4} \rtimes Q_{8}$, and the groups named in parts (iii)-(v) of Theorem 1.2.

Here $C_{4}$ and $Q_{8}$ stand for a cyclic group of order 4 and a quaternion group of order 8 , while $C_{4} \rtimes C_{4}$ and $C_{4} \rtimes Q_{8}$ indicate semidirect products which are not direct products (the last-named semidirect factor not being normal): in each of these two cases, there is only one isomorphism type of groups of this kind. Both groups satisfy condition (i) of Theorem 1.2.

It will be convenient to have a short temporary name for the 2 -groups $G$ such that if $g, h \in G$ then $\left\langle g^{2}\right\rangle$ is normal in $G$ and $\langle g, h\rangle /\left\langle g^{2}\right\rangle$ is either abelian or dihedral: let us call these groups $G$ good. Obviously, a group is good if and only if each of its two-generator subgroups is good, and so all subgroups of good groups are good. A little more thought shows that all factor groups of good groups are also good, and that the direct product of an elementary abelian 2-group with a good group is always good.

Of course, all abelian or dihedral 2 -groups are good. The next exploratory step is to look (for example, by using [6]) at each of the groups of order dividing 16 , and check that all but three of them are good. The three that fail do so because they are of the form $\langle g, h\rangle$ with $g^{2}=1$ but are neither abelian nor dihedral; they are $\left(C_{2} \times C_{2}\right) \rtimes C_{4}$ and the two semidirect products $C_{8} \rtimes C_{2}$ in which the action of $C_{2}$ on $C_{8}$ is neither 
trivial nor inverting. (The nonabelian $\left(C_{2} \times C_{2}\right) \rtimes C_{4}$ form a single isomorphism class.) We note for future use that the reasons which make the generalized quaternion group of order 16 good but the semidihedral group of order $16 \mathrm{bad}$, yield the same conclusions for generalized quaternion groups and semidihedral groups of larger orders as well.

Proof of the last sentence of Lemma 1.4. In case (i), all two-generator subgroups of $H$ are abelian or dihedral so $G$ is good. In cases (ii)-(v) we have $|\Phi(H)| \leq 4$, so the two-generator subgroups $K$ of $H$ are of order dividing 16 . No $K$ can be a $C_{8} \rtimes C_{2}$, because that would mean $\Phi(H) \geq \Phi(K) \cong C_{4}$ but $\Phi(H)$ contains no $C_{4}$. No $K$ can be a $\left(C_{2} \times C_{2}\right) \rtimes C_{4}$, because then $\Phi(H) \geq \Phi(K) \cong C_{2} \times C_{2}$ would exclude case (ii) and so (see Lemma 4.1) ensure $|\Omega(H)|=4$, contrary to $\Omega(K) \leq \Omega(H)$ and $|\Omega(K)|=8$. Thus in every case we may conclude that $G$ is good. This proves the converse part of Lemma 1.4.

The proof of the direct part needs more preparation. To avoid cumbersome circumlocution, we count $Q_{8}$ among the generalized quaternion groups. As usual, $x^{y}=y^{-1} x y$, and an involution is a group element of order 2 .

The first step is rather trivial, and the second is not much harder.

Lemma 4.2. If $H$ is a nonabelian good group with $|\Phi(H)|=2$ and no direct factor of order 2 , then it satisfies (ii) of Theorem 1.2.

Proof. Since $H$ has no direct factor of order 2, it has no central involution outside $\Phi(H)$; thus $|\Phi(H)|=2$ implies that $\mathrm{Z}(H)$ is cyclic of order at most 4 . If $|\mathrm{Z}(H)|=2$ then $H$ is extraspecial; otherwise there is a maximal subgroup $M$ which does not contain $\mathbf{Z}(H)$ and is easily seen to be extraspecial.

Lemma 4.3. An involution in a good group normalizes every cyclic subgroup of order greater than 2 and centralizes every subgroup isomorphic to $C_{4} \rtimes C_{4}$.

Proof. Let $g$ be an involution in a good group $G$. If $h$ is any element of $G$, then by the definition of 'good' $\langle g, h\rangle$ is abelian or dihedral: in either case, if $\langle h\rangle$ is of order greater than 2 then it is normalized by $g$. Suppose now that $x, y$ are elements of $G$ such that $\langle x, y\rangle \cong C_{4} \rtimes C_{4}$ with $x^{y}=x^{-1}$. If $x^{g}=x^{-1}$ and $y^{g}=y^{ \pm 1}$, then $g$ fails to normalize $\langle x y\rangle$, while if $x^{g}=x$ and $y^{g}=y^{-1}$, then $y g$ is an involution which does not normalize $\langle x y\rangle$ : so the only option is that $g$ centralizes $\langle x, y\rangle$.

We shall make repeated use of the fact that if $\langle x, y\rangle$ is a nonabelian dihedral 2 -group then $\langle x\rangle,\langle y\rangle$, $\langle x y\rangle$ are pairwise distinct and precisely two of them are non-normal subgroups of order 2 , while the third is normal and has order divisible by 4 .

If $\langle g, h\rangle$ is good and $\langle g\rangle$ is not normal in it, then $\langle g, h\rangle /\left\langle g^{2}\right\rangle$ is a nonabelian dihedral 2 -group, so it follows that $\left\langle g^{2}\right\rangle$ contains precisely one of $\left\langle h^{2}\right\rangle$ and $\left\langle(g h)^{2}\right\rangle$. Suppose further that neither $\langle h\rangle$ nor $\langle g h\rangle$ is normal in $\langle g, h\rangle$ : then, by this argument, each of $\left\langle g^{2}\right\rangle,\left\langle h^{2}\right\rangle$ and $\left\langle(g h)^{2}\right\rangle$ must contain one and only one of the other two. As it is impossible to order a three-element set in this manner, we have a contradiction, which proves that at least one of $\left\langle g^{2}\right\rangle,\left\langle h^{2}\right\rangle$ and $\left\langle(g h)^{2}\right\rangle$ must be normal in $\langle g, h\rangle$. We have proved that in a good group, every two-generator subgroup is metacyclic (in the sense of having a cyclic normal subgroup with cyclic quotient).

If a nonabelian 2-group has a cyclic normal subgroup of order 4 with cyclic quotient, then it is isomorphic to one of

$$
\begin{aligned}
& P_{k}=\left\langle w, y \mid w^{4}=1, w^{y}=w^{-1}, y^{2^{k}}=w^{2}\right\rangle, \\
& R_{k}=\left\langle w, y \mid w^{4}=1, w^{y}=w^{-1}, y^{2^{k+1}}=1\right\rangle .
\end{aligned}
$$

(This notation is not intended for use beyond the proof of the next lemma.) We claim that such a group is good if and only if $k \leq 1$. If $k \leq 1$ then both groups are of order dividing 16 and we have said nothing new. If $k>1$ then $P_{k}=\left\langle w y^{2^{k-1}}, y\right\rangle$ and $\left(w y^{2^{k-1}}\right)^{2}=1$ but $P_{k}$ is neither abelian nor dihedral and so cannot be good. As $P_{k}$ is a homomorphic image of $R_{k}$, in this case $R_{k}$ cannot be good either.

Lemma 4.4. If $\langle x, y\rangle$ is good and $\langle x\rangle$ is normal in it but $\langle y\rangle$ is not, then $|x| \geq 4 \geq|y|$ and $x^{y}=x^{-1}$. 
Proof. Since in $\langle x, y\rangle /\left\langle y^{2}\right\rangle$ the image of $\langle x\rangle$ is normal but the image of $\langle y\rangle$ is not, $\langle x, y\rangle /\left\langle y^{2}\right\rangle$ is a nonabelian dihedral group and the image of $\langle x\rangle$ has order divisible by 4 : thus

$$
x^{2} \notin\left\langle y^{2}\right\rangle
$$

and

$$
x^{y} \equiv x^{-1} \bmod \left\langle y^{2}\right\rangle \text {. }
$$

Of course (2) and the normality of $\langle x\rangle$ give that

$$
x^{y} \equiv x^{-1} \bmod \langle x\rangle \cap\left\langle y^{2}\right\rangle
$$

From (1) we know that $\langle x\rangle \cap\left\langle y^{2}\right\rangle \leq\left\langle x^{4}\right\rangle$, so by (3)

$$
x^{y} \equiv x^{-1} \bmod \left\langle x^{4}\right\rangle \text {. }
$$

It also follows from (1) that there is in $\langle x\rangle$ and element, $w$ say, of order 4 , and (4) implies that $w^{y}=w^{-1}$. Thus $\langle w, y\rangle$ is a $P_{k}$ or an $R_{k}$, and the argument leading up to the lemma may be invoked for the conclusion that $y^{4}=1$. If $y^{2} \notin\langle x\rangle$, then $\langle x\rangle \cap\left\langle y^{2}\right\rangle=1$ and so (3) gives that $x^{y}=x^{-1}$. If $y^{2} \in\langle x\rangle$, then $\langle x, y\rangle$ has a cyclic maximal subgroup and a nonabelian dihedral quotient, so it can only be dihedral or semidihedral or generalized quaternion. We have already seen that semidihedral groups are not good, so $x^{y}=x^{-1}$ holds in this case as well. Finally, $|x| \geq 4$ because $\langle x, y\rangle$ is nonabelian. This completes the proof of the lemma.

It follows that under the hypotheses of Lemma 4.4 the group $\langle x, y\rangle$ is either dihedral or generalized quaternion or a semidirect product $\langle x\rangle \rtimes\langle y\rangle$ with $|x| \geq|y|=4$ and $x^{y}=x^{-1}$.

Lemma 4.5. $f\langle x, u\rangle$ is good and both $\langle x\rangle$ and $\langle u\rangle$ are normal in it, then $\langle x, u\rangle$ is either abelian or isomorphic to $Q_{8}$.

Proof. We argue by contradiction. If the conclusion is false then $\langle x, u\rangle$ cannot be hamiltonian: so there is a cyclic subgroup, $\langle y\rangle$ say, which at least one of $x$ and $u$ fails to normalize. We may assume without loss of generality that $x$ does not normalize $\langle y\rangle$, and then Lemma 4.4 is conveniently applicable. At first we only use the conclusion that $x^{y}=x^{-1}$. By conjugation, $\langle x, u\rangle$ induces a cyclic group of automorphisms on $\langle x\rangle$, and now we know that this includes the inverting automorphism. In the automorphism group of a cyclic 2 -group, the subgroup generated by the inverting automorphism is a maximal cyclic subgroup ( -1 is not a square mod $2^{n}$ when $\left.n>1\right)$ : so the group induced by $\langle x, u\rangle$ is of order 2 . This proves that the centralizer $\mathrm{C}_{\langle x, u\rangle}(x)$, which contains $x$ but not $y$, is of index 2 . Thus $y \notin\langle x, \Phi(\langle x, u\rangle)\rangle$, and therefore $\langle x, y\rangle=\langle x, u\rangle$. However, of the groups of Lemma 4.4, only $Q_{8}$ can be generated by two normal cyclic subgroups, and we have assumed that $\langle x, u\rangle$ is not that group. This contradiction completes the proof of Lemma 4.5.

Since a good two-generator group is metacyclic, it has a generating set which satisfies the hypotheses of one of these two lemmas.

Corollary 4.6. A two-generator 2 -group is good if and only if it is either abelian or dihedral or generalized quaternion or a semidirect product $\langle x\rangle \rtimes\langle y\rangle$ with $|x| \geq|y|=4$ and $x^{y}=x^{-1}$.

Lemma 4.7. If $H$ is a nonabelian good group of exponent greater than 4, then $H$ satisfies (i) of Theorem 1.2 .

Proof. In the semidirect products of Corollary 4.6, all the elements outside the abelian group $\left\langle x, y^{2}\right\rangle$ have order 4 , and every cyclic subgroup of $\left\langle x, y^{2}\right\rangle$ is normal in $\langle x, y\rangle$. In a dihedral or generalized quaternion group which does not have exponent 4 , all cyclic subgroups of order greater than 4 are normal and lie in the unique cyclic maximal subgroup. It follows that in a good group every cyclic subgroup of order greater than 4 is normal and any two elements of order greater than 4 commute.

Let $A=\left\langle a \in H \mid a^{4} \neq 1\right\rangle$ : this is now an abelian subgroup of $H$. Let $a \in H$ with $a^{4} \neq 1$, and $h \in H$ but $h \notin A$, so $h^{4}=1$. Then $a$ and $h$ cannot commute (else $(a h)^{4} \neq 1$ and hence $a h \in A, h \in A$ would follow). Lemma 4.5 cannot apply with $x=a, u=h$, because $\langle a, h\rangle$ is neither abelian nor of exponent 4 . 
Hence Lemma 4.4 must apply with $x=a, y=h$. It follows that every element of $H$ outside $A$ must invert every element of $A$. If $H$ had more than one nontrivial coset modulo $A$, the quotient of two elements chosen from distinct nontrivial cosets would still lie outside $A$ : it would have to fix as well as invert every element of $A$. This being impossible, the index of $A$ in $H$ must be 2 .

Lemma 4.8. If $H$ is a good group of exponent 4, then its Frattini subgroup is elementary abelian and central.

Proof. If $g, h \in H$ then $\left[g^{2}, h\right]=1$ because, by Corollary $4.6,\langle g, h\rangle$ is either abelian or $D_{8}$ or $Q_{8}$ or $C_{4} \rtimes C_{4}$, and $\left[g^{2}, h\right]=1$ holds for every pair of elements $g, h$ in each of these groups. This shows that the Frattini subgroup is generated by central involutions.

In the proof of our next lemma, we shall make use of two properties of $C_{4} \rtimes C_{4}$. First, it has only two nontrivial elements that are squares. Second, as it can be generated by two non-commuting elements of order 4 whose product is also of order 4 , no automorphism of it can invert all elements of order 4 .

Lemma 4.9. If $a, x, y$ are elements of a good group $H$ of exponent 4 such that $x^{y}=x^{-1}$ and $a^{2} \notin\langle x, y\rangle \cong C_{4} \rtimes C_{4}$, then $x$ must centralize and $y$ must invert $a$. If also $b \in H$ and $a^{2} \neq b^{2} \notin\langle x, y\rangle$, then $a$ and $b$ commute.

Proof. The subgroup $\left\langle a^{2}\right\rangle$ is normal and the image of $\langle x, y\rangle$ in the quotient $H /\left\langle a^{2}\right\rangle$ is still a $C_{4} \rtimes C_{4}$. By Lemma 4.3, this image must centralize the image of $a$. It follows that $\langle x, y\rangle$ normalizes $\langle a\rangle$. It cannot centralize $a$, for then we would have $\langle a, x, y\rangle=\langle a\rangle \times\langle x, y\rangle$, and we know that $C_{4} \times\left(C_{4} \rtimes C_{4}\right)$ has a quotient $C_{4} \times D_{8}$ which is not good. Since $\langle x y, y\rangle=\langle x, y\rangle$, it follows that at least one of $x y$ and $y$ must invert $a$.

Suppose only one of them does: say, $a^{x y}=a^{-1}$ but $a^{y}=a$. Then $(a y)^{2} \notin\langle x, y\rangle$, and the above argument may be repeated with $a y$ in place of $a$, giving the conclusion that at least one of $x y$ and $y$ must invert $a y$. However, now $(a y)^{x y} \neq(a y)^{-1}$ because $a^{-1} x^{2} y \neq a^{-1} y^{-1}$, and $(a y)^{y}=a y \neq(a y)^{-1}$ : we have reached a contradiction. A similar argument gives a contradiction if we assume that $a^{x y}=a$ and $a^{y}=a^{-1}$. This proves that both $x y$ and $y$ must invert $a$, that is, $x$ must centralize and $y$ must invert $a$.

By Corollary 4.6, the only nonabelian good groups of exponent 4 generated by two elements of order 4 are $Q_{8}$ and $C_{4} \rtimes C_{4}$. Since $a^{2} \neq b^{2}$, we cannot have $\langle a, b\rangle \cong Q_{8}$. If $\langle a, b\rangle \cong C_{4} \rtimes C_{4}$, then $a^{2}$ and $b^{2}$ are the only nontrivial squares in $\langle a, b\rangle$, and by assumption neither of these lies in $\langle x, y\rangle$ : thus all cyclic subgroups of order 4 in $\langle a, b\rangle$ avoid $\langle x, y\rangle$ and are therefore inverted by $y$. Since no automorphism of $C_{4} \rtimes C_{4}$ can act like that, $a$ and $b$ must commute.

One of the two nontrivial squares in $C_{4} \rtimes C_{4}$ generates the commutator subgroup; hence if two cyclic subgroups of order 4 in $C_{4} \rtimes C_{4}$ intersect trivially, one of them must be normal. This will also be used in the proof of the next lemma.

In view of Lemma 4.8, if $H$ is a good group of exponent 4 then $\Phi(H)$ is an elementary abelian group spanned by squares, so it has a basis consisting of squares: that is, $H$ has a subset $X$ such that $\Phi(H)=\prod_{x \in X}\left\langle x^{2}\right\rangle$ and each $x^{2}$ is nontrivial.

Lemma 4.10. Let $H$ be a good group of exponent 4 and $X$ any subset such that $\Phi(H)=\prod_{x \in X}\left\langle x^{2}\right\rangle$ and each $x^{2}$ is nontrivial. Then either $\langle X\rangle$ is abelian or all but one of the elements of $X$ commute with each other and are inverted by the remaining one.

Proof. Suppose that $\langle X\rangle$ is nonabelian, and that $x, y$ is a noncommuting pair of elements of $X$. Since $\langle x\rangle \cap\langle y\rangle=1$, one of $\langle x\rangle$ and $\langle y\rangle$ normalizes the other: say, $x^{y}=x^{-1}$. By Lemma 4.9, then each element of $X \backslash\{x, y\}$ is centralized by $x$ and inverted by $y$, and any two elements of $X \backslash\{x, y\}$ commute with each other.

Lemma 4.11. If $H$ is a nonabelian good group of exponent 4, then the Frattini subgroup of its centre has order at most 2 . 
Proof. Suppose not: then $\mathrm{Z}(H)=\langle a\rangle \times\langle b\rangle \times \cdots$ with $|a|=|b|=4$. Since $H$ is nonabelian, it has a nonabelian two-generator subgroup $K$. By Corollary $4.6, K$ is either $D_{8}$ or $Q_{8}$ or $C_{4} \rtimes C_{4}$. We propose to show that $\langle a, b, K\rangle$ must contain a subgroup with a quotient isomorphic to $C_{4} \times D_{8}$. Since $C_{4} \times D_{8}$ has a subgroup $\left(C_{2} \times C_{2}\right) \rtimes C_{4}$ which we know is not good, this will contradict the assumption that $H$ is good, and so prove the lemma. If $\left\langle a^{2}, b^{2}\right\rangle \leq K$, we must have $K \cong C_{4} \rtimes C_{4}$, and then no generality is lost by assuming that $K=\left\langle x, y \mid x^{4}=y^{4}=1, x^{y}=x^{-1}\right\rangle$ and $a^{2}=x^{2}, b^{2}=y^{2}$, so $\langle a, a x, b y\rangle=\langle a\rangle \times\langle a x, b y\rangle=C_{4} \times D_{8}$. This argument tacitly involved changing $a$ and $b$ if necessary (without changing $\langle a, b\rangle$ ). The same flexibility allows us to assume that if $\left\langle a^{2}, b^{2}\right\rangle \not \leq K$ then $\langle a, b, K\rangle=\langle a\rangle \times\langle b, K\rangle$, and then what we need is that $D_{8}$ is a quotient of a subgroup of $\langle b, K\rangle$. Since $D_{8}$ is a quotient of $C_{4} \rtimes C_{4}$, this is only an issue if $K \cong Q_{8}$, but then the central product $C_{4} \mathrm{Y} Q_{8}$ is a quotient of $\langle b, K\rangle$ and of course $C_{4} \mathrm{Y} Q_{8} \cong C_{4} \mathrm{Y} D_{8}>D_{8}$.

Lemma 4.12. If $H$ is a nonabelian good group of exponent 4 with $|\Phi(H)|>4$, then $H$ satisfies (i) of Theorem 1.2 .

Proof. Let $X$ be a subset of $H$ of the kind discussed in Lemma 4.10.

First, suppose that $\langle X\rangle$ is abelian; then $\langle X\rangle=\prod_{x \in X}\langle x\rangle$. Set $A=\mathrm{C}(\langle X\rangle)$ : by Lemma 4.11, this centralizer is abelian. As $A$ is of exponent precisely 4 , it is generated by its elements of order 4 . Thus if an element $h$ normalizes every cyclic subgroup of order 4 in $A$ then it either centralizes or inverts $A$. If $h$ is an involution, then by Lemma 4.3 this comment is applicable. If we can show that each $h$ of order 4 acts on $A$ in this way, the claim of the lemma will follow.

Suppose then that $h \in H, h \notin A$, and $|h|=4$. If necessary, one can change $X$ without changing $\langle X\rangle$ (and therefore without changing $A$ ) so as to achieve that $X$ has an element, $x_{1}$ say, with $x_{1}^{2}=h^{2}$. Let $x_{2}$ be another element of $X$, and set $X^{\prime}=X \backslash\left\{x_{1}, x_{2}\right\}$. Then $\left\{h, x_{2}\right\} \cup X^{\prime}$ and $\left\{h, x_{1} x_{2}\right\} \cup X^{\prime}$ can also play the role of $X$ in Lemma 4.10. Since $X^{\prime}$ is nonempty (this is where we use the assumption that $|\Phi(H)|>4$ ) and commutes with $x_{2}$ and with $x_{1} x_{2}$, in each case $h$ is the only element which could invert all the others. Since $X^{\prime}$ is a nonempty common part of 'all the others', $h$ behaves the same way in both cases. If it centralizes in both cases, then it centralizes all of $X$, contrary to $h \notin A$. Thus $h$ inverts all elements of $\langle X\rangle$.

This proves that the centralizer $A$ of $X$ has at most one nontrivial coset in $H$, so $|H: A|$ is at most 2 . It cannot be 1 , because $A$ is abelian but $H$ is not. If $a$ is any element of order 4 in $A$, then there is an $x$ in $X$ such that for $Y=\{a\} \cup(X \backslash\{x\})$ we have $\Phi(H)=\prod_{y \in Y}\left\langle y^{2}\right\rangle$, so $Y$ can play the role of $X$ in all this. The centralizer of $Y$ contains and therefore equals $A$, so an element $h$ of order 4 outside $A$ inverts every element of $\langle Y\rangle$ as well. This proves that such an $h$ inverts every element of order 4 in $A$, and so it inverts every element of $A$, as required.

Second, suppose that $\langle X\rangle$ is not abelian: say, $y$ is the element of $X$ which inverts all the others, and all the others commute with each other. Set $X^{\prime}=X \backslash\{y\}$ and $A=\mathrm{C}\left(X^{\prime}\right)$. By Lemma 4.11, $A$ is abelian. If $\Phi(A)=\Phi(H)$, we can replace $X$ by a subset of $A$ and appeal to the half of the lemma which we have already proved. It remains to deal with the case $\Phi(A)<\Phi(H)$. Of course then $\Phi(A)=\Phi\left(\left\langle X^{\prime}\right\rangle\right)$, so $A$ is the direct product of $\left\langle X^{\prime}\right\rangle$ with an elementary abelian group. We shall show that every element $h$ outside $A$ inverts $A$.

Now $\langle x, y\rangle \cong C_{4} \rtimes C_{4}$ whenever $x \in X^{\prime}$, so Lemma 4.3 ensures that every involution lies in $A$ : we need only consider the $h$ of order 4 . If $h^{2} \notin\left\langle x^{2} \mid x \in X^{\prime}\right\rangle$ then $\{h\} \cup X^{\prime}$ can play the role of $X$ in Lemma 4.11; given that $X^{\prime}$ has at least two elements and they commute, this means that $h$ must either centralize or invert every element of $X^{\prime}$, that is, $h \in\langle y\rangle A$. If $h^{2} \in\left\langle x^{2} \mid x \in X^{\prime}\right\rangle$, one can change $X^{\prime}$ without changing $\left\langle X^{\prime}\right\rangle$ (and therefore without changing $A$ ) so as to achieve that $X^{\prime}$ has an element, $x_{1}$ say, with $x_{1}^{2}=h^{2}$. Let $x_{2}$ be another element of $X^{\prime}$ (here we use again the assumption that $|\Phi(H)|>4$ ), and set $X^{\prime \prime}=X^{\prime} \backslash\left\{x_{1}, x_{2}\right\}$. Both $\left\{y, h, x_{2}\right\} \cup X^{\prime \prime}$ and $\left\{y, h, x_{1} x_{2}\right\} \cup X^{\prime \prime}$ can play the role of $X$ in Lemma 4.11. Because $y$ inverts both $x_{2}$ and $x_{1} x_{2}$, we can conclude that $h$ commutes with $x_{2}$, with $x_{1} x_{2}$, and with every element of $X^{\prime \prime}$ (and is inverted by $y$ ). What matters is that in this case $h \in A$.

We have proved that $H=\langle y\rangle A$, and noted that $y$ centralizes, that is, inverts, every involution. We have also seen that $y$ inverts $\left\langle X^{\prime}\right\rangle$. Since $A$ is the direct product of $\left\langle X^{\prime}\right\rangle$ with an elementary abelian group, it follows that $y$ inverts $A$, and then so does every element of $H$ outside $A$. This completes the proof of the lemma. 
Lemma 4.13. If $h$ is a noncentral involution in a good group $H$ of exponent 4 with $|\Phi(H)|>2$, then $H$ has an abelian subgroup $A$ of index 2 such that every element of $A$ is inverted by $h$.

Proof. Since $h$ is noncentral it is noncentral already in some nonabelian two-generator subgroup which by Corollary 4.6 can only be dihedral: thus there is in $H$ an element $a$ of order 4 such that $a^{h}=a^{-1}$. Since $|\Phi(H)|>2$, there is also in $H$ an element $b$ such that $a^{2} \neq b^{2} \neq 1$. For any such $b$, by Corollary $4.6,\langle a, b\rangle$ is either abelian of a $C_{4} \rtimes C_{4}$. It cannot be the latter, because $h$ does not centralize it and we have Lemma 4.3. It follows that every such $b$ commutes with $a$. Further by Lemma $4.3, h$ must normalize both $\langle b\rangle$ and $\langle a b\rangle$, which is now only possible if $h$ inverts $b$. If $c \in H$ and $c^{2} \neq 1$, then either $c^{2} \neq a^{2}$ or $c^{2} \neq b^{2}$, and the above argument with $a, c$ or $b, c$ in place of $a, b$ yields that $h$ inverts $c$. We have proved that $h$ inverts every element of order 4 in $H$. Further, any two elements of order 4 commute: else by Corollary 4.6 the subgroup they generate would be a $Q_{8}$ or a $C_{4} \rtimes C_{4}$, and we have observed just before stating this lemma that neither of these groups has an automorphism that inverts all elements of order 4. Set $A=\left\langle a \in H \mid a^{2} \neq 1\right\rangle$; this is an abelian subgroup, and every element of it is inverted by $h$. If $g$ is an element of $H$ outside $A$, then $g$ is an involution (by the definition of $A$ ). If $\langle a, g\rangle$ were abelian, it would be generated by elements of order 4 and so would lie in $A$, contrary to $g \notin A$ : thus $g$ is a noncentral involution and, like $h$, inverts every element of $A$. Hence $g h$ centralizes $A$ and therefore cannot lie outside it. This means that every element $g$ of $H$ outside $A$ lies in the coset $A h$, that is, that $|H: A|=2$.

Proof of the direct part of Lemma 4.1. Let $G$ be a nonabelian good group. By Lemma 2.3, we may write $G$ as $E \times H$ with $E$ elementary abelian and $H$ having no direct factor of order 2, and of course $H$ is also nonabelian and good. If the exponent of $H$ is greater than 4, Lemma 4.7 shows that $H$ satisfies (i) of Theorem 1.2. Suppose that the exponent of $H$ is 4 . By Lemma 4.8, then $\Phi(H) \leq \mathrm{Z}(H)$. If $|\Phi(H)|=2$ then Lemma 4.2 shows that $H$ satisfies (ii), while if $|\Phi(H)|>4$ then (i) holds by Lemma 4.12. In the remaining case, $|\Phi(H)|=4$. If $H$ has a noncentral involution, (i) holds by Lemma 4.13. If all involutions are central, then $\Omega(H) \leq \Phi(H)$ because $H$ has no direct factor of order 2. We cannot have $|\Omega(H)|=2$, for then $H$ would be cyclic or generalized quaternion and (as $H$ has exponent 4) this is excluded by $|\Phi(H)|=4$. Thus $\Omega(H)=\Phi(H)$. Let $\Phi(H)=\left\langle g^{2}, h^{2}\right\rangle$ with $g, h \in H$, and $K$ any subgroup of $H$ which contains $\langle g, h\rangle$ : then also $\left\langle g^{2}, h^{2}\right\rangle=\Omega(K)=\Phi(K) \leq \mathrm{Z}(K)$ and so Lemma 4.1 (applied to $K$ in place of $H$ ) shows that $|K| \neq 128$. It follows that $H /\langle g, h\rangle$ is an elementary abelian 2 -group without a subgroup of order $128 / 16$; hence $H$ has order dividing 64 . Lemma 4.1 therefore shows that $H$ satisfies (i) or (iii) or (iv) or (v).

This completes the proof of Lemma 1.4, and so also the proof of Theorem 1.2.

The application of Lemma 4.1 to $K$ in place of $H$ raises a question: had the inspection of that CAYLEY library produced a different answer, would we still have a theorem? If the list of Lemma 4.1 did contain at least one group of order 128 , we could not save the present proof simply by adjusting the list in Theorem 1.2. However, instead of applying Lemma 4.1 to $K$ we could in any case appeal to Lemma 4.14 below, and so deduce that in the remaining case $H$ has order dividing 128. After that, even a Lemma 4.1 with a modified list would be good enough to conclude the proof (of a suitably modified theorem).

Lemma 4.14. If $H$ is a finite 2 -group such that $\Phi(H) \leq \Omega(H) \leq \mathrm{Z}(H)$ and $|\Omega(H)| \leq 2^{n}$, then $|H| \leq 2^{n(n+5) / 2}$.

Proof. If $n=0$ then $H=1$ while if $n=1$ then $H \leq Q_{8}$, so we have the initial step for a proof by induction on $n$. For the inductive step, suppose that $n>1$. If $H$ has exponent 2, the claim is obvious. Let $h$ be an element of order 4 in $H$. Since the conjugates of $h$ differ from $h$ by commutators and there are at most $2^{n}$ commutators in $H$, the centralizer $\mathrm{C}(h)$ has index at most $2^{n}$. As $\Phi(H)$ is of exponent 2 , it cannot contain $h$. Let $M$ be a maximal subgroup which does not contain $h$. Set $K=M \cap \mathrm{C}(h)$, and note that $|H: K| \leq 2^{n+1}$. If $h^{2}=k^{2}$ for some element $k$ of $K$, then $\left(h k^{-1}\right)^{2}=1$ and so $h k^{-1} \in \Omega(H)=\Phi(H) \leq M$ contradicts $h \notin M$. Thus all involutions of $K /\left\langle h^{2}\right\rangle$ lie in the group $\Omega(K) /\left\langle h^{2}\right\rangle$, and then by the inductive hypothesis $\left|K /\left\langle h^{2}\right\rangle\right| \leq 2^{(n-1)(n-1+5) / 2}$. Together with $|H: K| \leq 2^{n+1}$, this inequality gives the bound on $|H|$ that we wanted and so completes the inductive step.

At $n=2$, from Lemma 4.1 we can get $2^{6}$ where Lemma 4.14 gives $2^{7}$. Running the proof of Lemma 4.14 with this starting point, we can get an improved bound for higher values of $n$ as well. On the other hand, 
the direct product of $n$ copies of $Q_{8}$ shows that the $n(n+5) / 2$ in Lemma 4.14 could not be lowered below $3 n$. One may well wonder just what the optimal bound is.

\section{References}

1. A.A. Bovdi, Unitarity of the multiplicative group of an integral group ring. Mat. Sb. 119, 384-400 (1982) (Russian); English transl. in Math. USSR Sbornik 47, 377-389 (1984)

2. A. A. Bovdi, P. M. Gudivok, M. S. Semirot, Normal group rings. Ukrain. Mat. Zh. 37, 3-8 (1985) (Russian)

3. A. A. Bovdi and S. K. Sehgal, Unitary subgroups of integral group rings. Publ. Mat. 36, 197-204 (1992)

4. A. A. Bovdi and S. K. Sehgal, Unitary subgroups of integral group rings. Manuscripta Math. 76, 213-222 (1992)

5. V. A. Bovdi, Normal twisted group rings. Dokl. Akad. Nauk Ukrain. SSR (1990) No 7, 6-7 (Russian)

6. Marshall Hall, Jr. and James K. Senior, The groups of order $2^{n}(n \leq 6)$. Macmillan, New York, 1964

7. Graham Higman, Suzuki 2-groups. Illinois J. Math. 7, 79-96 (1963)

8. Irving Kaplansky, Infinite abelian groups. Revised edition, second printing; University of Michigan Press, Ann Arbor, 1971

9. M. F. Newman, On a class of nilpotent groups. Proc. London Math. Soc. (3) 10, 365-375 (1960)

10. M. F. Newman and E. A. O'Brien, A CAYLEY library for the groups of order dividing 128. Proceedings of the Singapore Group Theory Conference held at the National University of Singapore, 1987, ed. by Kai Nah CHENG and Yu Kiang Leong, de Gruyter, Berlin, New York, 1989; 437-442

11. Jürgen Ritter and Sudarshan K. Sehgal, Generators of subgroups of $U(Z G)$. Contemp. Math. 93, 331-347 (1989)

Victor Bovdi

Department of Mathematics

Bessenyei Teachers' College

4401 Nyíregyháza

Hungary

\section{G. Kovács}

School of Mathematical Sciences

Australian National University

Canberra ACT 0200

Australia

This article was processed by the authors using the Springer-Verlag TEX mamath macro package 1990. 